\title{
UNIQUE MINIMIZER FOR A RANDOM FUNCTIONAL WITH DOUBLE-WELL POTENTIAL IN DIMENSION 1 AND $2^{*}$
}

\author{
NICOLAS DIRR ${ }^{\dagger}$ AND ENZA ORLANDI ${ }^{\ddagger}$
}

\begin{abstract}
We add a random bulk term, modelling the interaction with the impurities of the medium, to a standard functional in the gradient theory of phase transitions consisting of a gradient term with a double well potential. We show that in $d \leq 2$ there exists, for almost all the realizations of the random bulk term, a unique random macroscopic minimizer. This result is in sharp contrast to the case when the random bulk term is absent. In the latter case there are two minimizers which are (in law) invariant under translations in space.
\end{abstract}

Key words. Random functionals, phase segregation in disordered materials.

AMS subject classifications. 35R60, 80M35, 82D30, 74Q05.

\section{Introduction}

Models where a stochastic contribution is added to the energy of the system naturally arise in condensed matter physics where the presence of the impurities causes the microscopic structure to vary from point to point.

We are interested in functionals which - without random perturbation - model the free energy of a material with two (or several) phases on a so called mesoscopic scale, i.e a scale which is much larger than the atomistic scale so that the adequate description of the state of the material is given by a continuous scalar order parameter $m: D \subseteq \mathbb{R}^{d} \rightarrow \mathbb{R}$. The minimizers of these functionals are functions $m^{*}$ representing the states or phases of the materials.

The natural question that we pose is the following: What happens to these minimizers when an external, even very weak, random force is added to the deterministic functional? Are there still the same number of minimizers, i.e will the material always have the same number of states (or phases)? Is there some significant difference in the qualitative properties of the material when the randomness is added? These are standard questions in a calculus of variations framework. However, standard techniques applicable for deterministic calculus of variation problems might not give a satisfactory answer when randomness is involved. In the case under consideration in this paper one needs to deal with a family of nonlinear functionals which are not convex and not bounded uniformly from below. So one needs to find, depending on the functionals, a way to answer these questions. It turns out that methods used in statistical mechanics, suitably modified, might give an answer to these problems in certain cases. Recently there has been an intensive effort, in both directions, to built a bridge between techniques and methods used in analysis and calculus of variations and those used in statistical mechanics; see, for a survey of these issues, [24]. This paper is in this context.

${ }^{*}$ Received: March 24, 2010; accepted (in revised version): August 10, 2010. Communicated by Jack Xin.

N.D. supported by GNFM-INDAM, E.O. supported by MURST/Cofin Prin07: 20078XYHYS and ROMA TRE University.

${ }^{\dagger}$ Department of Mathematical Sciences, University of Bath, Bath, BA2 7AY, United Kingdom (nd235@bath.ac.uk).

${ }^{\ddagger}$ Dipartimento di Matematica, Università di Roma Tre, L.go S. Murialdo 1, 00146 Roma, (orlandi@mat.uniroma3.it). 
The analysis of the asymptotic behavior of random functionals has received considerable attention within a homogenization framework; we mention for example the work by G. Dal Maso and L. Modica, [12, 13]. The techniques there are based on $\Gamma$-convergence from the analysis side and the sub-additive ergodic theorem from the probabilistic side.

More recently, A. Braides and A. Piatnitski [6] studied a random optimization problem, motivated by problems in mechanics, which requires techniques from percolation theory.

Problems from solid mechanics lead naturally to the mathematical analysis of the asymptotic behavior of random functionals; see e.g. [2, 3, 4].

The limit under consideration here, which requires techniques from statistical mechanics, is different from the problems mentioned previously due to two averaging effects taking places simultaneously: the singular limit of a functional with several ground states (minimizers), and the averaging over a random perturbation. The functional we will study here consists of three competing parts: an "interaction term" penalizing spatial changes in $m$, a double-well potential $W(m)$, i.e. a nonconvex function which has exactly two minimizers, for simplicity +1 and -1 , modelling a two-phase material, and a term which couples $m$ to a random field $\theta g(\cdot, \omega)$ with mean zero, variance $\theta^{2}$ and unit correlation length; i.e a term which prefers at each point in space one of the two minimizers of $W(\cdot)$ and breaks the translational invariance, but is "neutral" in the mean. A standard choice with the aforementioned properties is

$$
\hat{G}(m, \omega):=\int_{D}\left(|\nabla m(y)|^{2}+W(m(y))-\theta g(y, \omega) m(y)\right) \mathrm{dy} .
$$

We are, however, interested in a so-called macroscopic scale which is coarser than the mesoscopic scale. Therefore we rescale space with a small parameter $\epsilon$. If $\Lambda=\epsilon D$ and $u(x)=m\left(\epsilon^{-1} x\right)$ then we obtain $\hat{G}(m, \omega)=\epsilon^{1-d} G_{\epsilon}(u, \omega)$, where

$$
G_{\epsilon}(u, \omega, \Lambda):=\int_{\Lambda}\left(\epsilon|\nabla u(x)|^{2}+\frac{1}{\epsilon} W(m(x))-\frac{\theta}{\epsilon} g_{\epsilon}(x, \omega) m(x)\right) \mathrm{dx},
$$

and where $g_{\epsilon}$ has now correlation length $\epsilon$. We are interested in determining the minimizers of this functional, the asymptotic behavior (as $\epsilon \rightarrow 0)$ of them, and their qualitative properties.

Due to the non-convexity of the double-well potential, the Euler-Lagrange equation might not have a unique solution.

The $g$-dependent bulk term can, because of the scaling with $\epsilon^{-1}$, force a sequence $u_{\epsilon}$ to "follow" the oscillations of $g$. This always happens in the form of bounded oscillations around the two wells of the double well potential. In such a situation there are still two distinct minimizers. But in principle the $g$-dependent term could be strong enough to enforce large oscillations, so that the minimizers will "change well".

In the periodic case it is possible to check on a deterministic volume, with a diameter on the order of the period, whether the minimizer "changes well" (from one "well" of the double well potential to the other one) and so creates a "bubble" of the other phase. If it does not, then the symmetry of the double well implies immediately the existence of two distinct minimizers. If it changes from one well to the other, the situation is more complex, but can in principle be solved by considering a minimization problem on a period (i.e. a compact domain) with periodic boundary conditions. For further discussion of the periodic case we refer to $[7,14,15]$. 
The random case is quite different because there is no deterministic subset of $\Lambda$ such that the integral of the random field over this subset equals zero for almost all realizations of the random field - there are always fluctuations around the zero mean. A set $A$ becomes the support of a bubble of the other phase if the cost of switching to the other well, which can be estimated by the Modica-Mortola result (see [22] and [21]) as proportional to the boundary of $A$, is smaller than the integral of the random field part over $A$.

As the correlation length is $\epsilon$, a set $A \subseteq \Lambda$ contains roughly $|A| \epsilon^{-d}$ independent random variables, where $|\cdot|$ denotes the $d$ - dimensional Lebesgue measure of a set. By the central limit theorem, fluctuations of order $\theta \sqrt{|A|} \epsilon^{d / 2}$ are highly likely, but the probability of larger fluctuations vanishes exponentially fast. Therefore, using the isoperimetric inequality, the probability of $A$ being the support of a bubble is exponentially small if

$$
c_{d}|A|^{(d-1) / d} \gg|A|^{1 / 2} \epsilon^{(d-2) / 2} \theta
$$

where $c_{d}$ is the isoperimetric constant.

In $d \geq 3$ this is asymptotically always the case for sets of diameter of order larger than $\epsilon$, or for sets of any size, provided $\theta \rightarrow 0$. Dimension $d=2$ and $\theta$ small is a critical case. In $d \geq 3$, although (1.2) holds for one single bubble, to determine the properties of the minimizers one needs to ask if there exist "bubbles" of the other phase and of which sizes and how large they are. Notice that "bubbles" of the other phase with diameter of order $\epsilon$ will exist almost surely, if the system is large enough.

These kind of problems were discussed by the physics community in the 1980's for the random field Ising model. The problem was to determine the dimension at which the random field Ising model would show spontaneous magnetization at low temperature and weak disorder. The problem was solved by Bricmont and Kupiainen [8], who proved the existence of phase transition in $d \geq 3$ for small magnitude of the random field, and by Aizenman and Wehr [1], who proved that there is no phase transition in $d=2$ for all temperatures. Hence the case $d=3$ is indeed the physically critical case.

These kinds of problems are closely related to the question of whether there are at least two distinct minimizers, one predominantly + and one predominantly - , for functional (1.1).

This program for functional (1.1) has been successfully carried out in a previous paper by Dirr and Orlandi [16] for $d \geq 3$ and $\theta \simeq \frac{1}{|\log \epsilon|}$. They show that, $\mathbb{P}$ - a.s with respect to the random field, for any $\epsilon>0$ there were still two minimizers which, unlike in the case $\theta=0$, were not constant functions $u(x) \equiv 1$ and $u(x) \equiv-1$ but rather functions varying in $x$ and $\omega$, and the minimal energy was strictly negative. Further using the $\Gamma$-convergence technique they determined the cost of forming a bubble of one phase in the other one. These results were obtained under the strong assumption that $\theta \simeq \frac{1}{|\log \epsilon|}$. We expect, by analogy with the Ising models with random field, that, for $\theta$ small but fixed, in $d \geq 3$ there are still two minimizers but they do not stay in one single well, i.e one minimizer will be predominantly + and one predominantly -. In $d \geq 3$ for $\theta$ small the cost of creating a bubble of one phase located at a given point is larger than the possible gain of the fluctuations of the random field inside that bubble. So far there are no results for (1.1) in this case.

Here we address the case when $d \leq 2$ and the strength of the random field $\theta$ is fixed. We show that when $d=1,2$ there exists, for almost all the realizations of the random field, an unique macroscopic minimizer $u^{*}(\cdot, \omega)$ so that, denoting by $Q(0)$ the 
unit cube centered at the origin and by $Q(z)=z+Q(0)$ the unit cube centered in $z \in \mathbb{Z}^{d}$,

$$
\mathbb{E}\left[\int_{Q(z)} u^{*}(x, \cdot) d x\right]=0, \quad \forall z \in \mathbb{Z}^{d}, \quad d \leq 2,
$$

where $\mathbb{E}[\cdot]$ denotes the mean with respect to the random field. Note that for $\theta=0$ and for sufficiently small periodic forcing there exist two minimizers (see e.g.[15]), so the uniqueness of the minimizer is due to the random nature of the perturbation.

An explicit construction in 1-d is not easy, because if one observes an interval centered at the origin, it is not possible to give information about the local behavior of the minimizers of the functional on that interval without knowledge of the random field in a much larger random interval. But there is a related model - an Ising model with long-range (Kac type)interactions and a random field - where scaling limits of the 1-d minimizer have been characterized in a very specific way: In this case it is possible to show, via a suitable coarse graining, that when $\theta=0$ the size of intervals with positive or negative magnetization grows exponentially in $\frac{1}{T \gamma}$, where $\gamma^{-1}$ is the range of the interaction and the temperature $T$ is less than the critical mean field temperature $T_{c}$; see [11]. When the random field is added, i.e $\theta \neq 0$, the size of intervals with positive or negative magnetization goes algebraically as $\frac{1}{\gamma^{2}}$; see $[9,10]$ and $[23]$.

The strategy of our proof is based on the following steps. We prove first that there exist two macroscopic extremal minimizers $v^{ \pm}(\cdot, \omega)$ so that any other macroscopic minimizer satisfies $v^{-}(\cdot, \omega) \leq u^{*}(\cdot, \omega) \leq v^{+}(\cdot, \omega)$. By a standard argument we then show that for any $\Lambda \subset \mathbb{R}^{d}$ and for a positive constant $C$,

$$
\left|G_{1}\left(v^{+}, \omega, \Lambda\right)-G_{1}\left(v^{-}, \omega, \Lambda\right)\right| \leq C|\Lambda|^{\frac{d-1}{d}}, \quad \forall \omega \in \Omega .
$$

The minimizer $v^{ \pm}(\cdot, \omega)$ depends in a highly non trivial way on the random fields $g(x, \omega)$ for $x$ in all $\mathbb{Z}^{d}$. Therefore the difference $G_{1}\left(v^{+}, \omega, \Lambda\right)-G_{1}\left(v^{-}, \omega, \Lambda\right)$ depends on the random fields in all of $\mathbb{Z}^{d}$. We take a sequence $\Lambda_{n} \subset \Lambda_{n+1}$ and show that, conditioning on the random fields in $\Lambda_{n}$ (i.e taking the expectation over only the random fields outside $\Lambda_{n}$ ),

$$
F_{n}(\omega):=\mathbb{E}\left[G_{1}\left(v^{+}, \omega, \Lambda_{n}\right)-G_{1}\left(v^{-}, \omega, \Lambda_{n}\right) \mid \mathcal{B}_{\Lambda_{n}}\right]
$$

has significant fluctuations with variance of the order of the volume. The $\mathcal{B}_{\Lambda_{n}}$ is the $\sigma$-algebra generated by the random field in $\Lambda_{n}$. Namely we show that

$$
\mathbb{E}\left[F_{n}(\cdot)\right]=0,
$$

and

$$
\liminf _{n \rightarrow \infty} \mathbb{E}\left[e^{t \frac{F_{n}}{\sqrt{\Lambda_{n}}}}\right] \geq e^{\frac{t^{2} D^{2}}{2}},
$$

where $D^{2}$ is given in (4.51). This holds in all dimensions. But in $d \leq 2$ this generates a contradiction with the bound (1.3), unless $D^{2}=0$. When $D^{2}=0$ we show that $M=\mathbb{E}\left[\int_{Q(0)} v^{+}\right]-\mathbb{E}\left[\int_{Q(0)} v^{-}\right]=0$. Further, we show that $\mathbb{E}\left[\int_{Q(0)} v^{+}\right] \geq \mathbb{E}\left[\int_{Q(0)} v^{-}\right]$, and therefore $\mathbb{E}\left[\int_{Q(0)} v^{+}\right]=\mathbb{E}\left[\int_{Q(0)} v^{-}\right]=0$. The probabilistic argument has been already applied by Aizenman and Wehr [1] in the context of Ising spin systems with random external field; see also the book by Bovier [5] for a survey on this subject. 


\section{Notations and results}

2.1. The functional. The "macroscopic" space is given by $\Lambda:=\left[-\frac{1}{2}, \frac{1}{2}\right]^{d}$, the $d$ - dimensional unit cube centered at the origin. The ratio between the macroscopic and the "mesoscopic" scale is given by the small parameter $\epsilon$.

The disorder or random field is constructed with the help of a family of independent, identically distributed random variables with mean zero and variance equal to 1 . We assume that each random variable has distribution absolutely continuous with respect to the Lebesgue measure and that the Lebesgue density is a symmetric, compactly supported function on $\mathbb{R}$. The corresponding infinite product measure on $\mathbb{R}^{Z^{d}}$ will be denoted by $\mathbb{P}$ and by $\mathbb{E}[\cdot]$ the mean with respect to $\mathbb{P}$. In the following we will denote this family of random variables by $\{g(z, \omega)\}_{z \in \mathbb{Z}^{d}}, \omega \in \Omega$, where we identify $\Omega$ with $\mathbb{R}^{Z^{d}}$. These assumptions imply that there exists a finite $A>0$ so that

$$
\mathbb{E}[g(z)]=0, \quad \mathbb{E}\left[g^{2}(z)\right]=1, \quad \forall z \in \mathbb{Z}^{d} \quad \text { and } \quad\|g\|_{\infty}=\sup _{z}|g(z, \omega)|=A, \quad \mathbb{P} \text { - a.s. }
$$

The boundedness assumption is not essential. Different choices of $g$ could be handled by minor modifications provided $g$ is still a random field with finite correlation length, invariant under (integer) translations and such that $g(z, \cdot)$ has a symmetric distribution, absolutely continuous with respect to the Lebesgue measure and $\mathbb{E}\left[g(z)^{2+\eta}\right]<\infty$, $z \in \mathbb{Z}^{d}$ for $\eta>0$. The method does not apply when $g$ has atoms. In Ising spin systems, the uniqueness of the minimizer may fail if the distribution of $g$ has atoms; see [1]. The symmetry of the measure $\mathbb{P}$ is essential for obtaining the result, and not only for technical reasons: if $\mathbb{P}$ does not have a symmetric distribution, it would be no longer natural to compare the qualitative properties of the functional (1.1) with $\theta \neq 0$ with the functional (1.1) with $\theta=0$. (Note that in the case $\theta=0$ the functional is symmetric under the transformation $m \mapsto-m$.)

For example, if the mean of $g$ is no longer zero, one should compare the functional (1.1) with $\theta \neq 0$ with the functional (1.1) to which a term $h \int_{\Lambda} m(x) d x$ is added, with the constant $h$ equal to the mean of $g(z)$. In the following we always assume that $\mathbb{P}$ is symmetric.

We denote by $\mathcal{B}$ the product $\sigma$-algebra and by $\mathcal{B}_{\Lambda}, \Lambda \subset \mathbb{Z}^{d}$, the $\sigma$ - algebra generated by $\{g(z, \omega): z \in \Lambda\}$. In the following we often identify the random field $\left\{g(z, \cdot): z \in \mathbb{Z}^{d}\right\}$ with the coordinate maps $\left\{g(z, \omega)=\omega(z): z \in \mathbb{Z}^{d}\right\}$. To use ergodicity properties of the random field it is convenient to equip the probability space $(\Omega, \mathcal{B}, \mathbb{P})$ with some extra structure. First, we define the action $T$ of the translation group $\mathbb{Z}^{d}$ on $\Omega$. We will assume that $\mathbb{P}$ is invariant under this action and that the dynamical system $(\Omega, \mathcal{B}, \mathbb{P}, T)$ is stationary and ergodic. In our model the action of $T$ is, for $y \in \mathbb{Z}^{d}$,

$$
\left(g\left(z_{1},\left[T_{y} \omega\right]\right), \ldots, g\left(z_{n},\left[T_{y} \omega\right]\right)\right)=\left(g\left(z_{1}+y, \omega\right), \ldots, g\left(z_{n}+y, \omega\right)\right) .
$$

The disorder or random field in the functional will be obtained by a rescaling of $g$ such that the correlation length is order $\epsilon$ and the amplitude grows as $\epsilon \rightarrow 0$. To this end define for $x \in \Lambda$ a function $g_{\epsilon}(\cdot, \omega) \in L^{\infty}(\Lambda)$ by

$$
g_{\epsilon}(x, \omega):=\sum_{z \in \mathbb{Z}^{d}} g(z, \omega) \mathbb{1}_{\epsilon\left(z+\left[-\frac{1}{2}, \frac{1}{2}\right]^{d}\right) \cap \Lambda}(x),
$$

where for any Borel-measurable set $A$

$$
\mathbb{I}_{A}(x):=\left\{\begin{array}{l}
1, \text { if } x \in A, \\
0, \text { if } x \notin A .
\end{array}\right.
$$


The potential $W$ is a so-called "double-well potential":

Assumption (H1). $W \in C^{2}(\mathbb{R}), W \geq 0, W(s)=0$ iff $s \in\{-1,1\}, W(s)=W(-s)$, and $W(s)$ is strictly decreasing in $[0,1]$. Moreover, there exists $\delta_{0}$ and $C_{0}>0$ so that

$$
W(s)=\frac{1}{2 C_{0}}(s-1)^{2}, \quad \forall s \in\left(1-\delta_{0}, \infty\right) .
$$

Note that $W$ is slightly different from the standard choice $W(u)=\left(1-u^{2}\right)^{2}$. Our choice simplifies some proofs because it makes the Euler-Lagrange equation linear provided solutions stay in one "well". These assumptions could be relaxed. For $u \in H^{1}(\Lambda)$ and any open set $A \subseteq \Lambda$ consider the following random functional:

$$
G_{\epsilon}(u, \omega, A):=\int_{A}\left(\epsilon|\nabla u(x)|^{2}+\frac{1}{\epsilon} W(u(x))\right) \mathrm{d} x-\frac{1}{\epsilon} \theta \int_{A} g_{\epsilon}(x, \omega) u(x) \mathrm{dx},
$$

where $\theta>0$. Set $\epsilon=\frac{1}{n}, n \in \mathbb{N}$, so that for any $n \geq 1$ the mesoscopic space is defined as $\Lambda_{n}:=\left[-\frac{n}{2}, \frac{n}{2}\right]^{d}$. Consider $v \in H^{1}\left(\Lambda_{n}\right)$ and denote in mesoscopic coordinates

$$
G_{1}\left(v, \omega, \Lambda_{n}\right):=\int_{\Lambda_{n}}\left(|\nabla v(x)|^{2}+W(v(x))\right) \mathrm{dx}-\theta \int_{\Lambda_{\mathrm{n}}} \mathrm{g}_{1}(\mathrm{x}, \omega) \mathrm{v}(\mathrm{x}) \mathrm{dx} .
$$

The relation between (2.5) and (2.6) is

$$
G_{n}(u, \omega, \Lambda)=n^{-(d-1)} G_{1}\left(v, \omega, \Lambda_{n}\right),
$$

where $v(x)=u\left(\frac{1}{n} x\right)$ for $x \in \Lambda_{n}$.

For $n>1$ fixed and $\omega \in \Omega$ it follows in the same way as in the case without random perturbation that the functional $G_{1}(\cdot, \omega)$ is coercive and weakly lower semicontinuous in $H^{1}\left(\Lambda_{n}\right)$, so there exists at least one minimizer (see [17]) which is a random function in $H^{1}\left(\Lambda_{n}\right)$; i.e. different realizations of $\omega$ will give different minimizers.

Definition 2.1. Translational covariant states. We say that the function $v$ : $\mathbb{R}^{d} \times \Omega \rightarrow \mathbb{R}$ is translational covariant if

$$
v(x+y, \omega)=v\left(x,\left[T_{-y} \omega\right]\right) \quad \forall y \in \mathbb{Z}^{d}, \quad x \in \mathbb{R}^{d} .
$$

2.2. Minimizers. Our main result is the following.

\section{THEOREM 2.2 .}

Take $d \leq 2, \theta$ strictly positive and $u_{n}^{*}(\cdot, \omega) \in \operatorname{argmin}_{w \in H^{1}\left(\Lambda_{n}\right)} G_{1}\left(w, \omega, \Lambda_{n}\right)$. Then, $\mathbb{P}$ a.s. there exists an unique $u^{*}(\cdot, \omega)$ defined as

$$
\left.\lim _{n \rightarrow \infty} u_{n}^{*}(x, \omega)=u^{*}(\cdot, \omega) \quad \text { (uniformly on compacts in } x\right),
$$

so that

- $u^{*}(\cdot, \omega)$ is translation covariant (see $(2.8)$ ),

- $u^{*}(\cdot, \omega)$ is Lipschitz continuous in $\mathbb{R}^{d}$,

- $\left|u^{*}(\cdot, \omega)\right| \leq 1+C_{0} \theta\left\|g_{1}\right\|_{\infty}$ where $C_{0}$ is the constant in (2.4),

$$
\lim n^{-d} G_{1}\left(u_{n}^{*}(\cdot, \omega), \omega, \Lambda_{n}\right)=\lim n^{-d}\left(\inf _{H^{1}\left(\Lambda_{n}\right)} G_{1}\left(\cdot, \omega, \Lambda_{n}\right)\right)=e,
$$

where $e$ is a deterministic value given in (4.13), and 


$$
\mathbb{E}\left[\int_{z+\left[-\frac{1}{2}, \frac{1}{2}\right]^{d}} u^{*}(x, \omega) d x\right]=0, \quad \forall z \in \mathbb{Z}^{d} .
$$

REMARK 2.3. When $\theta=0$ in (2.6), i.e the random field is absent, the minimum value is zero and there are two minimizers: the constant functions identical equal to 1 or to -1 .

REMARK 2.4. In the case analyzed in $[16], d \geq 3, \theta \simeq \frac{\tilde{\theta}}{\log n}, \tilde{\theta} \in(0,1)$, there exist two minimizers

$$
u^{ \pm}(\cdot, \omega)= \pm 1+v^{*}(\cdot, \omega), \quad \mathbb{E}\left[v^{*}(x, \cdot)\right]=0, \quad \sup _{x}\left|v^{*}(x, \omega)\right| \leq C_{0} \tilde{\theta}\|g\|_{\infty} .
$$

\section{Finite volume minimizers}

In this section we state properties for minimizers of the following problem

$$
\min _{w \in H^{1}\left(\Lambda_{n}\right)} G_{1}\left(w, \omega, \Lambda_{n}\right) .
$$

These properties hold in all dimensions $d$ and for almost every $\omega \in \Omega$. The volume $\Lambda_{n}$ is kept fixed throughout the section. Thus to shorten notation we denote $\Lambda:=\Lambda_{n}$ and state the results for any $d$ while $\omega$ plays the role of a parameter. We first show that to determine the minimizers of the functional $G_{1}$ it is sufficient to consider functions in $H^{1}(\Lambda)$ which satisfy a uniform $L^{\infty}$-bound:

Lemma 3.1. Assume (H1). For all $\omega \in \Omega$, for all $v \in H^{1}(\Lambda)$, and all $t>1+C_{0} \theta\|g\|_{\infty}$,

$$
G_{1}(v, \omega, \Lambda)-G_{1}(t \wedge(v \vee(-t)), \omega, \Lambda) \geq \int_{\Lambda_{t}}\left(C_{0}^{-1}(t-1)-\theta\|g\|_{\infty}\right)(|v(y)|-t),
$$

where $C_{0}$ is the constant in (2.4) and $\Lambda_{t}=\{y \in \Lambda:|v(y)|>t\}$. In particular $G_{1}(t \wedge v \vee$ $(-t), \omega, \Lambda)<G_{1}(v, \omega, \Lambda)$ unless $\Lambda_{t}=\emptyset$.

Proof.

$$
\begin{aligned}
G_{1}(v, \omega, \Lambda)- & G_{1}(t \wedge v \vee(-t), \omega, \Lambda) \\
& \geq \int_{\Lambda_{t}}(W(v(y))-W(t)) d y-\theta \int_{\Lambda_{t}} g_{1}(y, \omega)[v(y)-\operatorname{sign}(v(y)) t] d y,
\end{aligned}
$$

and from (H1) and the $L^{\infty}$-bound on $g$ we derive (3.1).

This $L^{\infty}$ bound on the global minimizer implies Lipschitz-regularity. Namely, a minimizer of $G_{1}(\cdot, \omega)$ in $H^{1}(\Lambda)$ is a weak solution of the Euler-Lagrange equation

$$
\begin{cases}\Delta v=\frac{1}{2}\left[W^{\prime}(v)+\theta g_{1}\right] & \text { in } \Lambda, \quad \omega \in \Omega, \\ \frac{\partial v}{\partial n}=0 & \text { on } \partial \Lambda .\end{cases}
$$

We have the following regularity result.

Proposition 3.2. Let $v(\cdot, \omega)$ be a solution of the Euler-Lagrange equation (3.2) and set

$$
L_{0}=C(d)\left[\sup _{\{s: s=v(r), r \in \Lambda\}}\left|W^{\prime}(s)\right|+\theta\|g\|_{\infty}\right],
$$


where $C(d)$ is a positive constant which depends on the dimension d. We have

$$
\left|v(r, \omega)-v\left(r^{\prime}, \omega\right)\right|<L_{0}\left|r-r^{\prime}\right|, \quad r, r^{\prime} \in \Lambda, \quad \forall \omega \in \Omega .
$$

Proof. $\quad$ By Lemma 3.1, a global minimizer $v$ satisfies the bound $|v(r, \omega)| \leq$ $1+C_{0} \theta\|g\|_{\infty}$ for $r \in \Lambda$ and $\omega \in \Omega$. Since $\left|g_{1}(\cdot, \omega)\right| \leq A$ for all $\omega \in \Omega$ (see (2.1)), any minimizer will be a bounded solution of Poisson's equation with a bounded right hand side.

By the regularity theory for the Laplacian (see [18]) the solution $v$ is Lipschitz in $\Lambda$ with a Lipschitz constant bounded by the quantity $L_{0}$ defined in (3.3).

The following lemma proves that minimizers of $G_{1}(\cdot, \omega, \Lambda)$ corresponding to ordered boundary conditions on $\Lambda$ are ordered as well; i.e they do not intersect. In particular if there exists more than one minimizer corresponding to the same boundary condition then they do not intersect.

Lemma 3.3. Let $w_{1}$ and $w_{2}$ be functions in $H^{1}(\Lambda)$ such that (in the sense of traces) $w_{1} \leq w_{2}$ on $\partial \Lambda$, and

$$
u \in \operatorname{argmin}_{w-w_{1} \in H_{0}^{1}(\Lambda)} G_{1}(w, \omega, \Lambda) \quad \text { and } \quad v \in \operatorname{argmin}_{w-w_{2} \in H_{0}^{1}(\Lambda)} G_{1}(w, \omega, \Lambda) .
$$

Then $u=v$ or $|u(x)-v(x)|>0$ for all $x \in \operatorname{int}(\Lambda)$. If $w_{1}<w_{2}$ in an open set in $\partial \Lambda$, then $u<v$ everywhere in $\operatorname{int}(\Lambda)$.

Proof. The argument works for general functionals of the type

$$
E(w):=\int_{\Lambda}\left(|\operatorname{grad} w(x)|^{2}+f(w, x)\right) d x,
$$

where $\partial_{w w} f(w, x)$ is continuous on $\mathbb{R} \times \bar{\Lambda}$ (here we treat $\omega$ as parameter, i.e. it holds for any realization of the random field).

Note that for any $H^{1}$-functions $u$ and $v$,

$$
E(u \vee v)+E(u \wedge v)=E(u)+E(v) .
$$

If $u \in \operatorname{argmin}_{w-w_{1} \in H_{0}^{1}(\Lambda)} E(w)$ and $v \in \operatorname{argmin}_{w-w_{2} \in H_{0}^{1}(\Lambda)} E(w)$ we have $u \vee v=v, u \wedge$ $v=u$ on $\partial \Lambda$. By the minimization properties of $u$ and $v$ we get $E(u \vee v) \geq E(v), E(u \wedge$ $v) \geq E(u)$. This implies that actually $E(u \vee v)=E(v), E(u \wedge v)=E(u)$, so $u \vee v \in$ $\operatorname{argmin}_{w-w_{2} \in H_{0}^{1}} E(u), u \wedge v \in \operatorname{argmin}_{w-w_{1} \in H_{0}^{1}} E(u)$. Obviously the function $m:=u-$ $u \wedge v \geq 0$ in $\Lambda$ and in particular $m=0$ on $\partial \Lambda$. We have that $m$ in our context solves

$$
\begin{aligned}
& \Delta m=\frac{1}{2}\left[f^{\prime}(u)-f^{\prime}(u \wedge v)\right]=V(x) m \quad \text { in } \Lambda, \\
& m=0 \quad \text { on } \quad \partial \Lambda
\end{aligned}
$$

with potential

$$
V(x)=\frac{1}{2} \frac{f^{\prime}(u)-f^{\prime}(u \wedge v)}{u-u \wedge v},
$$

which is continuous because $f$ is twice continuously differentiable in its first argument.

Suppose there exists $x_{0} \in \Lambda$ with $m\left(x_{0}\right)=0$. By Harnack's inequality (see [18], Theorem 8.20) for nonnegative solutions to elliptic linear equations, $\sup _{B_{R}\left(x_{0}\right)} m \leq$ 
$C \inf _{B_{R}\left(x_{0}\right)} m$ for any ball such that $B_{4 R} \subset \Lambda$. The constant $C>0$ depends on the radius $R$ and the coefficients in (3.4). Hence $0 \leq m \leq \sup _{B_{R}\left(x_{0}\right)} m=0$, so $m \equiv 0$ on such a ball. It immediately follows that $m \equiv 0$ on int $(\Lambda)$. Therefore in the interior of $\Lambda$ either $u=u \wedge v$ (in which case $u \leq v$ ) or $u>u \wedge v$; i.e. $v<u$. As minimizers are uniformly Lipschitz continuous, the latter case is only possible if $u=v$ on $\partial \Lambda$.

Consider the first case: $\widehat{m}:=v-u \geq 0$. We get, reasoning as before, $\Delta \widehat{m}=\widehat{V}(x) \widehat{m}$ with a uniformly continuous potential $\widehat{V}$. Then arguing as above $\widehat{m}=0$ everywhere or $\widehat{m}>0$ everywhere.

\section{Infinite volume covariant states}

THEOREM 4.1. [infinite-volume states] For almost all $\omega \in \Omega$ there exist two functions $v^{+}(x, \omega), v^{-}(x, \omega), x \in \mathbb{R}^{d}$, having the following properties:

- $v^{ \pm}(\cdot, \omega)$ is Lipschitz continuous in $\mathbb{R}^{d}$,

$$
\left|v^{ \pm}(\cdot, \omega)\right| \leq 1+C_{0} \theta\left\|g_{1}\right\|_{\infty},
$$

where $C_{0}$ is the constant in (2.4),

$$
v^{+}(x, \omega)=-v^{-}(x,-\omega) \quad x \in \mathbb{R}^{d},
$$

- $v^{ \pm}(\cdot, \omega)$ are translation covariant,

$$
\bullet
$$

$$
\lim n^{-d} \int_{\Lambda_{n}} v^{ \pm}(x, \omega) \mathrm{d} x=m^{ \pm}
$$

- where $m^{ \pm}=\mathbb{E}\left[\int_{\left[-\frac{1}{2}, \frac{1}{2}\right] d} v^{ \pm}(x, \cdot) \mathrm{d} x\right]$, and $m^{+}=-m^{-} \geq 0$,

$$
\lim n^{-d} G_{1}\left(v^{+}, \omega, \Lambda_{n}\right)=\lim n^{-d} G_{1}\left(v^{-}, \omega, \Lambda_{n}\right)=\lim n^{-d} \inf _{H^{1}\left(\Lambda_{n}\right)} G_{1}\left(\cdot, \omega, \Lambda_{n}\right)=e
$$

where $e$ is deterministic value given in (4.13), and

- If $\bar{w}_{n}(\cdot, \omega) \in \operatorname{argmin}_{H^{1}\left(\Lambda_{n}\right)} G_{1}\left(v, \omega, \Lambda_{n}\right)$ then

$$
v^{-}(x, \omega) \leq \liminf _{n \rightarrow \infty} \bar{w}_{n}(x, \omega) \leq \limsup _{n \rightarrow \infty} \bar{w}_{n}(x, \omega) \leq v^{+}(x, \omega), \quad x \in \mathbb{R}^{d} .
$$

Proof. We start proving the existence. Consider the following boundary problems. For $z \in \mathbb{Z}^{d}, C=C_{0}\|g\|_{\infty}$ where $C_{0}$ defined in (2.4),

$$
\begin{aligned}
& \inf _{(v-(1+C \theta)) \in H_{0}^{1}\left(\Lambda_{n}+z\right)} G_{1}\left(v, \omega, z+\Lambda_{n}\right), \\
& \inf _{(v+1+C \theta) \in H_{0}^{1}\left(\Lambda_{n}+z\right)} G_{1}\left(v, \omega, z+\Lambda_{n}\right) .
\end{aligned}
$$

Denote by $v_{n}^{z,+}:=v_{n}^{z,+}(\cdot, \omega)$ the maximal minimizer of (4.6) and by $v_{n}^{z,-}:=v_{n}^{z,-}(\cdot, \omega)$ the minimal minimizer of (4.7). If $z=0$ we write $v_{n}^{ \pm}$. For each $n>0$ and for each $\omega \in \Omega$ there exists at least one minimizer of problems (4.6) and (4.7) by lower semicontinuity and coerciveness. By Lemma 3.1, $v_{m}^{+} \leq 1+C \theta$ on $\partial \Lambda_{n}$ for $m>n$. Lemma 3.3 implies 
that for any $x$ and $\omega$ (and $\left.n>n_{0}(x)\right)$ the sequence $\left\{v_{n}^{+}(x)\right\}_{n}$ is decreasing. Moreover it is bounded from below by $-1-C \theta$. Hence, reasoning in a similar manner for $v_{n}^{-}$,

$$
v^{ \pm}(x, \omega):=\lim _{n} v_{n}^{ \pm}(x, \omega)
$$

exists and is measurable as a function of $\omega$. As the $v_{n}^{ \pm}$are bounded and minimizers, they are uniformly bounded and uniformly Lipschitz on each fixed cube $A$ which does not depend on $n$; see Proposition 3.2. This implies that subsequences converge locally uniformly to a Lipschitz function. As the entire sequence converges pointwise, the limit of any subsequence must coincide with $v^{ \pm}$, which is therefore Lipschitz. The same argument for general $z$ yields monotone limits $v^{z, \pm}$.

To show (4.2) we note that

$$
\begin{aligned}
\inf _{(v-(1+C \theta)) \in H^{1}\left(\Lambda_{n}\right)} G_{1}\left(v, \omega, \Lambda_{n}\right) & =\inf _{(v-(1+C \theta)) \in H^{1}\left(\Lambda_{n}\right)} G_{1}\left(-v,-\omega, \Lambda_{n}\right) \\
& =\inf _{(w+(1+C \theta)) \in H^{1}\left(\Lambda_{n}\right)} G_{1}\left(w,-\omega, \Lambda_{n}\right) .
\end{aligned}
$$

If $\bar{v}(\cdot, \omega) \in \operatorname{argmin}_{(v-(1+C \theta)) \in H_{0}^{1}} G_{1}\left(v, \omega, \Lambda_{n}\right)$ the function

$$
-\bar{v}(\cdot, \omega)=\bar{w}(\cdot,-\omega) \in \operatorname{argmin}_{\left.(w+(1+C \theta)) \in H_{0}^{1}\right)} G_{1}\left(w,-\omega, \Lambda_{n}\right),
$$

so that $\bar{v}(\cdot, \omega)=-\bar{w}(\cdot,-\omega)$. Therefore if $\bar{v}(\cdot, \omega)$ is the maximal minimizer of $\inf _{(v-(1+C \theta)) \in H_{0}^{1}} G_{1}\left(v, \omega, \Lambda_{n}\right)$, then $\bar{w}(\cdot,-\omega)$ is the minimal minimizer of $\inf _{(w+(1+C \theta)) \in H_{0}^{1}} G_{1}\left(w,-\omega, \Lambda_{n}\right)$.

To show the translation covariance, i.e. $v^{0,+}(0, \omega)=v^{z,+}\left(z, T_{-z} \omega\right)$, we notice that, by $(2.2)$,

$$
v_{n}^{0,+}(0, \omega)=v_{n}^{z,+}\left(z, T_{-z} \omega\right) .
$$

As the limit does not depend on the subsequence, we know that $v^{+}=\lim v_{2^{n}}^{+}$. As for $n$ large $\Lambda_{n}+z \subseteq \Lambda_{2^{n}}$, we get $v_{n}^{z,+}(0) \leq v_{2^{n}}^{0,+}(0)$ and $v^{z,+}(0) \leq v^{0,+}(0)$. The opposite inequality follows in the same way.

Next we want to show (4.3). We have

$$
\begin{aligned}
& \int_{\Lambda_{n}} v^{ \pm}(x, \omega) \mathrm{d} x=\sum_{z \in \Lambda_{n} \cap \mathbb{Z}^{d}} \int_{\left\{z+\left[-\frac{1}{2}, \frac{1}{2}\right]^{d}\right\}} v^{ \pm}(x, \omega) \mathrm{d} x \\
= & \sum_{z \in \Lambda_{n} \cap \mathbb{Z}^{d}} \int_{\left[-\frac{1}{2}, \frac{1}{2}\right]^{d}} v^{ \pm}\left(T_{z} x, \omega\right) \mathrm{d} x=\sum_{z \in \Lambda_{n} \cap \mathbb{Z}^{d}} \int_{\left[-\frac{1}{2}, \frac{1}{2}\right]^{d}} v^{ \pm}\left(x, T_{-z} \omega\right) \mathrm{d} x .
\end{aligned}
$$

Since $\left|v^{ \pm}(x, \omega)\right| \leq C$, by Birkhoff's ergodic theorem (see for example [20]) we have $\mathbb{P}$ a.s

$$
\begin{aligned}
& \lim \frac{1}{n^{d}} \int_{\Lambda_{n}} v^{ \pm}(x, \omega) \mathrm{d} x=\lim \frac{1}{n^{d}} \sum_{z \in \Lambda_{n} \cap \mathbb{Z}^{d}} \int_{\left[-\frac{1}{2}, \frac{1}{2}\right]^{d}} v^{ \pm}\left(x, T_{-z} \omega\right) \mathrm{d} x \\
= & \mathbb{E}\left[\int_{\left[-\frac{1}{2}, \frac{1}{2}\right]^{d}} v^{ \pm}(x, \cdot) \mathrm{d} x\right]=m^{ \pm} .
\end{aligned}
$$

Next we show (4.4). By the covariance property of $v^{ \pm}(\cdot, \cdot)$ and the choice of the double 
well potential $W(W$ does not depend on $x)$ we have

$$
\begin{aligned}
G_{1}\left(v^{+}(\omega), \omega, \Lambda_{n}\right) & =\sum_{z \in \Lambda_{n} \cap \mathbb{Z}^{d}} G_{1}\left(v^{+}(\omega), \omega, z+\left[-\frac{1}{2}, \frac{1}{2}\right]^{d}\right) \\
& =\sum_{z \in \Lambda_{n} \cap \mathbb{Z}^{d}} G_{1}\left(v^{+}\left(T_{-z} \omega\right), T_{-z} \omega,\left[-\frac{1}{2}, \frac{1}{2}\right]^{d}\right) .
\end{aligned}
$$

Therefore, by Birkhoff's ergodic theorem, $\mathbb{P}-$ a.s

$$
\lim \frac{1}{n^{d}} G_{1}\left(v^{+}(\omega), \omega, \Lambda_{n}\right)=\mathbb{E}\left[G_{1}\left(v^{+}(\cdot), \cdot\left[-\frac{1}{2}, \frac{1}{2}\right]^{d}\right)\right] .
$$

Since

$$
G_{1}\left(v^{+}(\omega), \omega, \Lambda_{n}\right)=G_{1}\left(-v^{+}(\omega),-\omega, \Lambda_{n}\right)=G_{1}\left(v^{-}(-\omega),-\omega, \Lambda_{n}\right)
$$

we have

$$
\mathbb{E}\left[G_{1}\left(v^{+}(\cdot), \cdot\left[-\frac{1}{2}, \frac{1}{2}\right]^{d}\right)\right]=\mathbb{E}\left[G_{1}\left(v^{-}(\cdot), \cdot\left[-\frac{1}{2}, \frac{1}{2}\right]^{d}\right)\right]=e .
$$

To show the last equality of (4.4) note that if $\bar{w}_{n}(\cdot, \omega) \in \operatorname{argmin}_{H^{1}\left(\Lambda_{n}\right)} G_{1}\left(\cdot, \omega, \Lambda_{n}\right)$ then

$$
G_{1}\left(\bar{w}_{n}, \omega, \Lambda_{n}\right) \leq G_{1}\left(v_{n}^{+}, \omega, \Lambda_{n}\right) .
$$

Moreover, let the cut-off function $\Psi: \mathbb{R} \rightarrow \mathbb{R}$ be nondecreasing, 1-Lipschitz and such that $\Psi(x)=0$ for $x<0, \Psi(x)=1$ for $x>2$. Then

$$
\hat{w}_{n}:=\Psi\left(\operatorname{dist}\left(x, \mathbb{R}^{d} \backslash \Lambda_{n}\right)\right) \bar{w}_{n}+\left(1-\Psi\left(\operatorname{dist}\left(x, \mathbb{R}^{d} \backslash \Lambda_{n}\right)\right)\right) v_{n}^{+}
$$

satisfies the boundary conditions of $v_{n}^{+}$, hence

$$
G_{1}\left(\hat{w}_{n}, \omega, \Lambda_{n}\right) \geq G_{1}\left(v_{n}^{+}, \omega, \Lambda_{n}\right) .
$$

Moreover an explicit calculation using the Lipschitz bounds of the minimizers, $\Psi$, and the double well potential together with the bounds on the random field shows that

$$
G_{1}\left(\hat{w}_{n}, \omega, \Lambda_{n}\right) \leq G_{1}\left(\bar{w}_{n}, \omega, \Lambda_{n}\right)+C n^{d-1}, \quad \forall \omega \in \Omega,
$$

where $C>0$ depends only on the double well potential and on the bound on the random field. (For details see proof of Lemma 4.2.)

Taking (4.14)-(4.16) together, we obtain that $\lim \frac{1}{n^{d}} G_{1}\left(\bar{w}_{n}, \omega, \Lambda_{n}\right)=$ $\lim \frac{1}{n^{d}} G_{1}\left(v_{n}^{+}, \omega, \Lambda_{n}\right)$. It remains to show (4.5). Let $x, w_{n}$ be as in the statement, and $n$ large enough so that $x \in \Lambda_{n}$. Note that by Lemma 3.1, $v_{n}^{-}(y, \omega) \leq w_{n}(y, \omega) \leq v_{n}^{+}(y, \omega)$ for all $y \in \partial \Lambda_{n}$. So by Lemma 3.3 we get that $v_{n}^{-}(x, \omega) \leq w_{n}(x, \omega) \leq v_{n}^{+}(x, \omega)$. (4.5) follows by taking liminf and limsup.

Next we bound uniformly in $\omega$ the difference between the energy of the maximal + minimizer and the minimal - minimizer.

Lemma 4.2. Let $\omega \in \Omega, \quad u^{+} \in \operatorname{argmin}_{v-\left(1+C_{0} A \theta\right) \in H_{0}(\Lambda)} G_{1}(v, \omega, \Lambda), \quad u^{-} \in$ $\operatorname{argmin}_{v+\left(1+C_{0} A \theta\right) \in H_{0}(\Lambda)} G_{1}(v, \omega, \Lambda)$, where $C_{0}$, and $A$ are the constants respectively in (2.4) and (2.1). There exists a positive constant $C$ depending on $\theta, C_{0}$, and A so that

$$
\left|G_{1}\left(u^{+}, \omega, \Lambda\right)-G_{1}\left(u^{-}, \omega, \Lambda\right)\right| \leq C|\Lambda|^{\frac{d-1}{d}} .
$$


Proof. Set

$$
\tilde{u}(x, \omega)=\left\{\begin{array}{l}
u^{+}(x, \omega) \quad \text { for } \quad x \in \Lambda \backslash\{x \in \Lambda: d(x, \partial \Lambda) \leq 1\} \\
u(x), \quad u(x)+\left(1+C_{0} A \theta\right) \in H_{0}^{1}(\Lambda), \quad x \in \Lambda: d(x, \partial \Lambda) \leq 1,
\end{array}\right.
$$

where $u$ is an arbitrary Lipschitz function, with $|\nabla u(x)| \leq 2\left(1+C_{0} A \theta\right)$, chosen to match the boundary conditions, i.e $\tilde{u}(x, \omega)+\left(1+C_{0} A \theta\right) \in H_{0}^{1}(\Lambda)$. We have

$$
\begin{aligned}
G_{1}(\tilde{u}, \omega, \Lambda)= & \int_{\{x \in \Lambda: d(x, \partial \Lambda) \leq 1\}}\left[\left(|\nabla \tilde{u}(x)|^{2}+W(\tilde{u}(x))\right)-\left(\left|\nabla u^{+}(x)\right|^{2}+W\left(u^{+}(x)\right)\right)\right] \mathrm{dx} \\
& +G_{1}\left(u^{+}, \omega, \Lambda\right)+\theta \int_{\{x \in \Lambda: d(x, \partial \Lambda) \leq 1\}} g_{1}(x, \omega)\left[\tilde{u}(x)-u^{+}(x)\right] \mathrm{dx} \\
\leq & G_{1}\left(u^{+}, \omega\right)+|\Lambda|^{\frac{d-1}{d}}\left[C+4 \theta\left\|g_{1}\right\|_{\infty}\right],
\end{aligned}
$$

where $C=C\left(C_{0}, \theta, A\right)$ is a positive constant which might change from one occurrence to the other. Obviously

$$
G_{1}\left(u^{-}, \omega, \Lambda\right) \leq G_{1}(\tilde{u}, \omega, \Lambda)
$$

Therefore

$$
G_{1}\left(u^{-}, \omega, \Lambda\right)-G_{1}\left(u^{+}, \omega, \Lambda\right) \leq|\Lambda|^{\frac{d-1}{d}}\left[C+4 \theta\left\|g_{1}\right\|_{\infty}\right] .
$$

Similarly one can show that

$$
G_{1}\left(u^{+}, \omega, \Lambda\right)-G_{1}\left(u^{-}, \omega, \Lambda\right) \leq|\Lambda|^{\frac{d-1}{d}}\left[C+4 \theta\left\|g_{1}\right\|_{\infty}\right] .
$$

Therefore (4.17).

The quantity next defined plays a fundamental role.

Definition 4.3. Let $v^{ \pm}(\omega)$ be the infinite volume states constructed in Theorem 4.1. Denote

$$
F_{n}(\omega):=\mathbb{E}\left[G_{1}\left(v^{+}(\omega), \omega, \Lambda_{n}\right)-G_{1}\left(v^{-}(\omega), \omega, \Lambda_{n}\right) \mid \mathcal{B}_{\Lambda_{n}}\right]
$$

REMARK 4.4. By definition $F_{n}(\cdot)$ is $\mathcal{B}_{\Lambda_{n}}$ measurable and by the symmetry assumption on the random field $\left\{g(z, \cdot), z \in \mathbb{Z}^{d}\right\}$,

$$
\mathbb{E}\left[F_{n}(\cdot)\right]=0
$$

Namely $v^{+}(x, \omega)=-v^{-}(x,-\omega)$ for $x \in \mathbb{R}^{d}$. This implies that

$$
G_{1}\left(v^{+}(\omega), \omega, \Lambda_{n}\right)=G_{1}\left(v^{-}(-\omega),-\omega, \Lambda_{n}\right)
$$

and by the symmetry of the random field we get (4.21).

Next we want to quantify how much $v^{ \pm}(\omega)$ changes when the random field is modified only in one site, for example at the site $i$. We introduce the following notation:

$$
\omega^{(i)}: \omega^{(i)}(z)=\omega(z) \quad z \neq i, \quad \omega=\left(\omega(i), \omega^{(i)}\right) \quad i, z \in \mathbb{Z}^{d}
$$


Then $v^{+}\left(\cdot,\left(\omega(0), \omega^{(0)}\right)\right)$ is the state $v^{+}$when the random field at the origin is $\omega(0)$, and $v^{+}\left(\cdot,\left(\omega(0)-h, \omega^{(0)}\right)\right)$ the state $v^{+}$when the random field at the origin is $\omega(0)-h$. Same definition for the infinite volume state $v^{-}\left(\cdot,\left(\cdot, \omega^{(0)}\right)\right)$ and for the finite volume minimizers $v_{n}^{ \pm}\left(\cdot,\left(\cdot, \omega^{(0)}\right)\right)$.

We have the following:

Lemma 4.5. For $\Lambda \subset \mathbb{R}^{d}, 0 \in \Lambda, h>0$ we have

$$
\begin{aligned}
& \theta h \int_{Q(0)} v^{+}\left(\omega(0), \omega^{(0)}\right) \mathrm{d} x \\
\geq & G_{1}\left(v^{+}\left(\omega(0)-h, \omega^{(0)}\right),\left(\omega(0)-h, \omega^{(0)}\right), \Lambda\right)-G_{1}\left(v^{+}\left(\omega(0), \omega^{(0)}\right),\left(\omega(0), \omega^{(0)}\right), \Lambda\right) \\
\geq & \theta h \int_{Q(0)} v^{+}\left(\omega(0)-h, \omega^{(0)}\right) \mathrm{d} x,
\end{aligned}
$$

where $Q(0):=[-1 / 2,1 / 2]^{d}$. The same inequalities hold for $v^{-}$.

Proof. Let $\Lambda_{n}$ be a cube centered at the origin so that $\Lambda \subset \Lambda_{n}$. Let $v_{n}^{+}$be the maximal minimizer of

$$
\inf _{(v-(1+C \theta)) \in H_{0}^{1}\left(\Lambda_{n}\right)} G_{1}\left(v, \omega, \Lambda_{n}\right) .
$$

Note that $v_{n}^{+}$is measurable with respect to the random field $g(z, \omega), z \in \Lambda_{n} \cap \mathbb{Z}^{d}$. We have

$$
\begin{aligned}
& G_{1}\left(v_{n}^{+}\left(\omega(0), \omega^{(0)}\right),\left(\omega(0), \omega^{(0)}\right), \Lambda\right)-G_{1}\left(v_{n}^{+}\left(\omega(0)-h, \omega^{(0)}\right),\left(\omega(0)-h, \omega^{(0)}\right), \Lambda\right) \\
= & G_{1}\left(v_{n}^{+}\left(\omega(0), \omega^{(0)}\right),\left(\omega(0), \omega^{(0)}\right), \Lambda\right)-G_{1}\left(v_{n}^{+}\left(\omega(0), \omega^{(0)}\right),\left(\omega(0)-h, \omega^{(0)}\right), \Lambda\right) \\
& +G_{1}\left(v_{n}^{+}\left(\omega(0), \omega^{(0)}\right),\left(\omega(0)-h, \omega^{(0)}\right), \Lambda\right)-G_{1}\left(v_{n}^{+}\left(\omega(0)-h, \omega^{(0)}\right),\left(\omega(0)-h, \omega^{(0)}\right), \Lambda\right) .
\end{aligned}
$$

By explicit computation (see (2.6)), we have that

$$
\begin{aligned}
& G_{1}\left(v_{n}^{+}\left(\omega(0), \omega^{(0)}\right),\left(\omega(0), \omega^{(0)}\right), \Lambda\right)-G_{1}\left(v_{n}^{+}\left(\omega(0), \omega^{(0)}\right),\left(\omega(0)-h, \omega^{(0)}\right), \Lambda\right) \\
= & -h \theta \int_{Q(0)} v_{n}^{+}\left(\omega(0), \omega^{(0)}\right) d x .
\end{aligned}
$$

The last line in (4.25) is nonnegative, because $v_{n}^{+}\left(g(0)-h, \omega^{(0)}\right)$ is a minimizer of (4.24) when the random field is $\left(g(0)-h, \omega^{(0)}\right)$. Therefore

$$
\begin{aligned}
& G_{1}\left(v_{n}^{+}\left(\omega(0)-h, \omega^{(0)}\right),\left(\omega(0)-h, \omega^{(0)}\right), \Lambda\right)-G_{1}\left(v_{n}^{+}\left(\omega(0), \omega^{(0)}\right),\left(\omega(0), \omega^{(0)}\right), \Lambda\right) \\
\leq & h \theta \int_{Q(0)} v_{n}^{+}\left(\omega(0), \omega^{(0)}\right) d x .
\end{aligned}
$$

By splitting

$$
\begin{aligned}
& G_{1}\left(v_{n}^{+}\left(\omega(0), \omega^{(0)}\right),\left(\omega(0), \omega^{(0)}\right), \Lambda\right)-G_{1}\left(v_{n}^{+}\left(\omega(0)-h, \omega^{(0)}\right),\left(\omega(0)-h, \omega^{(0)}\right), \Lambda\right) \\
= & G_{1}\left(v_{n}^{+}\left(\omega(0), \omega^{(0)}\right),\left(\omega(0), \omega^{(0)}\right), \Lambda\right)-G_{1}\left(v_{n}^{+}\left(\omega(0)-h, \omega^{(0)}\right),\left(\omega(0), \omega^{(0)}\right), \Lambda\right) \\
& +G_{1}\left(v_{n}^{+}\left(\omega(0)-h, \omega^{(0)}\right),\left(\omega(0), \omega^{(0)}\right), \Lambda\right)-G_{1}\left(v_{n}^{+}\left(\omega(0)-h, \omega^{(0)}\right),\left(\omega(0)-h, \omega^{(0)}\right), \Lambda\right),
\end{aligned}
$$


we obtain in a similar way

$$
\begin{aligned}
& G_{1}\left(v_{n}^{+}\left(\omega(0)-h, \omega^{(0)}\right),\left(\omega(0)-h, \omega^{(0)}\right), \Lambda\right)-G_{1}\left(v_{n}^{+}\left(\omega(0), \omega^{(0)}\right),\left(\omega(0), \omega^{(0)}\right), \Lambda\right) \\
\geq & h \theta \int_{Q(0)} v_{n}^{+}\left(\omega(0)-h, \omega^{(0)}\right) d x .
\end{aligned}
$$

To pass to the limit note that the cube $Q(0)$ remains fixed. Denote by $M$ the smallest integer such that $\Lambda \subseteq B_{M}(0)$, where $B_{M}(0)$ is a ball centered at the origin of radius $M$. Let $\xi(r)$ for $r \geq 0$ be a smooth cut-off function such that $\xi(r)=1$ for $r<M, \xi=0$ for $r>2 M$. Note that for $n>2 \operatorname{diam}(\Lambda)$ the function

$$
\hat{v}_{n}^{+}:=v_{n}^{+} \xi\left(|x|^{2}\right)
$$

satisfies

$$
\Delta \hat{v}_{n}^{+}=f_{n}(x)
$$

with $\sup _{n}\left\|f_{n}\right\|_{\infty}<C, C$ depending on $\operatorname{diam}(\Lambda), \theta$, the double well potential, the cutoff function and the bound $\|g\|_{\infty}$ on the random field. The first derivatives of $\hat{v}_{n}^{+}$are, away from the boundary, Hölder continuous with any exponent $\alpha<1$ (take $\alpha=1 / 2$ for definiteness) and the Hölder norm bounded uniformly in $n$ with a bound depending only on $C$. (See [18], Theorem 3.9. Note that this interior estimate is applicable because our domain is a ball containing $2 \Lambda$, so the square $\Lambda$ is contained in the interior.) So an application of the Arzela-Ascoli Theorem gives that for a subsequence $v_{n}^{+}$and $\operatorname{grad} v_{n}^{-}$converge uniformly. By Lebesgue's Theorem on dominated convergence, we may pass to the limit under the integral and the claim is shown.

The corresponding statements for $v^{-}$are proved in the same way.

REMARK 4.6. From Lemma 4.5 we have that

$$
\omega(0) \mapsto \int_{Q(0)} v^{+}\left(\omega(0), \omega^{(0)}\right) \mathrm{d} x
$$

is nondecreasing.

COROLlary 4.7. Let $\omega(i)$ be the random field in the site $i$ which has probability distribution absolutely continuous with respect to the Lebesgue measure. We have that $G_{1}\left(v^{+}(\omega), \omega, \Lambda\right)$ is $\mathbb{P}$-a.e. differentiable with respect to $\omega(i)$ and

$$
\frac{\partial G_{1}\left(v^{ \pm}(\omega), \omega, \Lambda\right)}{\partial \omega(i)}=-\theta \int_{Q(i)} v^{ \pm}(x, \omega) \mathrm{d} x
$$

Proof. It is sufficient to consider the case $i=0$. By applying Lemma 4.5 for $\omega(0)$ and $\tilde{\omega}(0):=\omega(0)+h$ we see that left and right derivatives exist and are equal if $s \mapsto \int_{Q(0)} v^{+}\left(s, \omega^{(0)}\right) \mathrm{d} x$ is continuous at $s=\omega(0)$. By Remark 4.6 this happens for Lebesgue almost all $s$, and hence by the assumptions on the random field $\mathbb{P}$-a.e.

THEOREM 4.8. We have that

$$
\lim _{n \rightarrow \infty} \frac{1}{\sqrt{\left|\Lambda_{n}\right|}}\left[F_{n}(\cdot)\right] \stackrel{D}{=} Z
$$

where $Z$ stands for a Gaussian random variable with mean 0 and variance $b^{2}$ with

$$
4 \theta^{2}\left(1+C_{0} \theta\|g\|_{\infty}\right)^{2} \geq b^{2} \geq \mathbb{E}\left[\left(\mathbb{E}\left[F_{n} \mid \mathcal{B}(0)\right]\right)^{2}\right],
$$


where $\mathcal{B}(0)$ is the $\sigma$-algebra generated by $g(0, \omega)$ and $C_{0}$ is given in (2.4).

Proof. We prove the theorem invoking the general result presented in the appendix. In order to do so, we need to establish the relevant conditions. We decompose $F_{n}$ as a martingale difference sequence. We order the points in $\Lambda_{n} \cap \mathbb{Z}^{d}$ according to the lexicographic ordering. In the following $i \leq j$ refers to the lexicographic ordering. Any other ordering will be fine but it is convenient to fix one. We introduce the family of increasing $\sigma$-algebras $\mathcal{B}_{n, i}, i \in \Lambda_{n} \cap \mathbb{Z}^{d}$ where $\mathcal{B}_{n, i}$ is the $\sigma$-algebra generated by the random variables $\left\{g(z), z \in \Lambda_{n} \cap \mathbb{Z}^{d}, z \leq i\right\}$. We denote by

$$
\mathcal{B}_{n, 0}=(\emptyset, \Omega), \quad \mathcal{B}_{n, i} \subset \mathcal{B}_{n, j} \quad i \leq j, \quad i \in \Lambda_{n} \cap \mathbb{Z}^{d}, \quad j \in \Lambda_{n} \cap \mathbb{Z}^{d} .
$$

We split

$$
F_{n}=\sum_{i \in \mathbb{Z}^{d} \cap \Lambda_{n}}\left(\mathbb{E}\left[F_{n} \mid \mathcal{B}_{n, i}\right]-\mathbb{E}\left[F_{n} \mid \mathcal{B}_{n, i-1}\right]\right):=\sum_{i \in \mathbb{Z}^{d} \cap \Lambda_{n}} Y_{n, i}
$$

By construction $\mathbb{E}\left[Y_{n, i}\right]=0$ for $i \in \mathbb{Z}^{d} \cap \Lambda_{n}$, and $\mathbb{E}\left[Y_{n, i} \mid \mathcal{B}_{n, k}\right]=0$ for all $0 \leq k \leq i-1$. Denote

$$
V_{n}:=\frac{1}{\left|\Lambda_{n} \cap \mathbb{Z}^{d}\right|} \sum_{i \in \Lambda_{n} \cap \mathbb{Z}^{d}} \mathbb{E}\left[Y_{n, i}^{2} \mid \mathcal{B}_{n, i-1}\right]
$$

In Lemma 4.9 stated and proven below we show that $V_{n} \rightarrow b^{2}$ in probability and $b^{2}$ satisfies (4.27). In Lemma 4.10 stated and proven below we show that for any $a>0$,

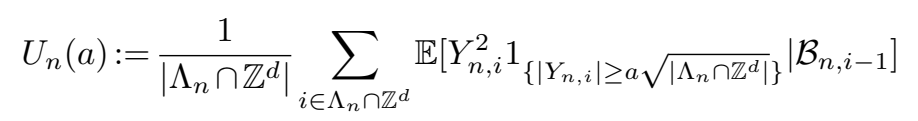

converges to 0 in probability. We can then invoke Theorem 5.1, stated in the appendix. The correspondence to the notation used in the appendix is the following. Identify $\left|\Lambda_{n} \cap \mathbb{Z}^{d}\right|$ with $n, \frac{F_{n}}{\sqrt{\left|\Lambda_{n} \cap \mathbb{Z}^{d}\right|}} \leftrightarrow S_{n}, \frac{Y_{n, i}}{\sqrt{\left|\Lambda_{n} \cap \mathbb{Z}^{d}\right|}} \leftrightarrow X_{n, i}$ and $\mathcal{B}_{n, i} \leftrightarrow \mathcal{F}_{n, i}$. Then (4.26) is obtained.

Lemma 4.9. Let $V_{n}$ be the quantity defined in (4.29). For all $\delta>0$,

$$
\lim _{n \rightarrow \infty} \mathbb{P}\left[\left|V_{n}-b^{2}\right| \geq \delta\right]=0
$$

where for $W_{0}$ is defined in (4.34), and

$$
b^{2}=\mathbb{E}\left[W_{0}^{2}\right]
$$

Furthermore

$$
4 \theta^{2}\left(1+C_{0} \theta\|g\|_{\infty}\right)^{2} \geq b^{2} \geq \mathbb{E}\left[\left(\mathbb{E}\left[F_{n} \mid \mathcal{B}(0)\right]\right)^{2}\right]
$$

where $C_{0}$ is given in (2.4).

Proof. The proof of (4.31) is done by applying conveniently the ergodic theorem. We introduce new $\sigma$-algebra $\mathcal{B}_{i}^{\leq}$generated by the random fields $\left\{g(z, \omega), z \in \mathbb{Z}^{d}, z \leq i\right\}$, where $\leq$ refers to the lexicographic ordering. Define for $i \in \Lambda_{n}$

$$
\begin{aligned}
W_{i}[\omega]= & \mathbb{E}\left[G_{1}\left(v^{+}(\omega), \omega, \Lambda_{n}\right)-G_{1}\left(v^{-}(\omega), \omega, \Lambda_{n}\right) \mid \mathcal{B}_{i}^{\leq}\right] \\
& -\mathbb{E}\left[G_{1}\left(v^{+}(\omega), \omega, \Lambda_{n}\right)-G_{1}\left(v^{-}(\omega), \omega, \Lambda_{n}\right) \mid \mathcal{B}_{i-1}^{\leq}\right] .
\end{aligned}
$$


Note that $W_{i}$ is a random variable depending on random fields on sites smaller or equal than $i$ under the lexicographic order. In particular it does not depend on the choice of the cube $\Lambda_{n}$ provided $i \in \Lambda_{n}$. To verify this statement notice that $v^{ \pm}(\omega)$ does not depend on $\Lambda_{n}$. Further, denote for $i \in \Lambda_{n}, \omega=\left(\omega_{i}^{<}, \omega(i), \omega_{i}^{>}\right)$where $\omega_{i}^{<}=(\omega(j), j<i)$ and $\omega_{i}^{>}=(\omega(j), j>i), \tilde{\omega}=\left(\omega_{i}^{<}, \tilde{\omega}(i), \omega_{i}^{>}\right)$and $\omega(s)=\left(\omega_{i}^{<}, s, \omega_{i}^{>}\right), s \in[-1,1]$. We can write $W_{i}[\omega]$ as

$$
\begin{aligned}
W_{i}[\omega]= & \int \mathbb{P}\left(d \omega_{i}^{>}\right) \mathbb{P}(d \tilde{\omega}(i))\left[G_{1}\left(v^{+}(\omega), \omega, \Lambda_{n}\right)-G_{1}\left(v^{+}(\tilde{\omega}), \tilde{\omega}, \Lambda_{n}\right)\right] \\
& -\int \mathbb{P}\left(d \omega_{i}^{>}\right) \mathbb{P}(d \tilde{\omega}(i))\left[G_{1}\left(v^{-}(\omega), \omega, \Lambda_{n}\right)-G_{1}\left(v^{-}(\tilde{\omega}), \tilde{\omega}, \Lambda_{n}\right)\right]
\end{aligned}
$$

By Corollary 4.7, $G_{1}\left(v^{+}(\omega), \omega, \Lambda_{n}\right)$ is a.e. differentiable with respect to $\omega(i)$ with derivative depending only on the random field on $Q(i)$. Therefore one has

$$
\begin{aligned}
G_{1}\left(v^{+}(\omega), \omega, \Lambda_{n}\right)-G_{1}\left(v^{+}(\tilde{\omega}), \tilde{\omega}, \Lambda_{n}\right) & =\int_{\tilde{\omega}(i)}^{\omega(i)} \frac{\partial}{\partial s} G_{1}\left(v^{+}(\omega(s)), \omega(s), \Lambda_{n}\right) d s \\
& =-\theta \int_{Q(i)} d x \int_{\tilde{\omega}(i)}^{\omega(i)} v^{+}\left(x, \omega^{<}, s, \omega^{>}\right) d s .
\end{aligned}
$$

Similar considerations hold for the last term of (4.35). Hence $W_{i}$ does not depend on the choice of $\Lambda_{n}$, provided $Q(i) \subset \Lambda_{n},{ }^{1}$.

Note that from the translation covariant properties of $v^{ \pm}$we have

$$
W_{i}[\omega]=W_{0}\left[T_{-i} \omega\right]
$$

By construction (see (4.28)), for any $i$ provided $n$ large enough so that $i \in \Lambda_{n}$, we have

$$
Y_{n, i}=\mathbb{E}\left[W_{i} \mid \mathcal{B}_{\Lambda_{n}}\right]
$$

Furthermore, by Corollary 4.7

$$
\left|W_{0}(\omega)\right| \leq 2 \theta\left(1+C_{0} \theta\|g\|_{\infty}\right), \quad \omega \in \Omega,
$$

where $C_{0}$ is given in (2.4). Applying the ergodic theorem we have that in probability

$$
\lim _{n \rightarrow \infty} \frac{1}{\left|\Lambda_{n} \cap \mathbb{Z}^{d}\right|} \sum_{i \in \Lambda_{n} \cap \mathbb{Z}^{d}} \mathbb{E}\left[W_{i}^{2} \mid \mathcal{B}_{i-1}^{\leq}\right]=\mathbb{E}\left[W_{0}^{2}\right] .
$$

Set $\mathbb{E}\left[W_{0}^{2}\right]=b^{2}$. Recalling the definition of $V_{n}$ given in (4.29), the proof of (4.31) is completed if we show the following: For any $\delta>0$,

$$
\lim _{n \rightarrow \infty} \mathbb{P}\left[\left|\mathbb{E}\left[Y_{n, i}^{2} \mid \mathcal{B}_{n, i-1}\right]-\mathbb{E}\left[W_{i}^{2}|| \mathcal{B}_{i-1}^{\leq}\right]\right| \geq \delta\right]=0 .
$$

We show (4.40) applying Chebyshev's inequality. We split

$$
\begin{aligned}
& \left\{\mathbb{E}\left[Y_{n, i}^{2} \mid \mathcal{B}_{n, i-1}\right]-\mathbb{E}\left[W_{i}^{2} \mid \mathcal{B}_{i-1}^{\leq}\right]\right\} \\
= & \mathbb{E}\left[Y_{n, i}^{2}-W_{i}^{2} \mid \mathcal{B}_{n, i-1}\right]+\mathbb{E}\left[W_{i}^{2} \mid \mathcal{B}_{n, i-1}\right]-\mathbb{E}\left[W_{i}^{2}|| \mathcal{B}_{i-1}^{\leq}\right] .
\end{aligned}
$$

\footnotetext{
${ }^{1}$ When the distribution of the random field is not absolutely continuous with respect to the Lebesgue measure we are able to prove that the difference in (4.36) has lower and upper bounds which do not depend on $\Lambda_{n}$. This does not imply that the difference itself does not depend on $\Lambda_{n}$.
} 
Denote $f_{i}=\mathbb{E}\left[W_{i}^{2} \mid \mathcal{B}_{i-1}^{\leq}\right], R_{n}=R_{n}(i)=\operatorname{dist}\left(i, \partial \Lambda_{n}\right)$ and $\mathcal{B}_{i+\left[-R_{n}, R_{n}\right]^{d}}$ the $\sigma-$ algebra generated by the random fields in the box $\left[-R_{n}, R_{n}\right]^{d}$ centered in $i$. We have

$$
\mathbb{E}\left[\left(\mathbb{E}\left[W_{i}^{2} \mid \mathcal{B}_{n, i-1}\right]-\mathbb{E}\left[W_{i}^{2}|| \mathcal{B}_{i-1}^{\leq}\right]\right)^{2}\right] \leq \mathbb{E}\left[\left(f_{0}-E\left[f_{0} \mid \mathcal{B}_{\left[-R_{n}, R_{n}\right]}\right]\right)^{2}\right]=: b_{1}\left(R_{n}\right) .
$$

When $\lim _{n \rightarrow \infty} R_{n}=\infty$, we have for any square integrable function

$$
\lim _{n \rightarrow \infty} b_{1}\left(R_{n}\right)=0 \text {. }
$$

Furthermore, by (4.37) we have

$$
\mathbb{E}\left[\left|\mathbb{E}\left[Y_{n, i}^{2}-W_{i}^{2} \mid \mathcal{B}_{n, i-1}\right]\right|\right] \leq \mathbb{E}\left[\left|Y_{n, 0}^{2}-W_{0}^{2}\right|\right] \leq\left(E\left[W_{0}^{2}\right]\right)^{\frac{1}{2}}\left(E\left[\left(Y_{n, 0}-W_{0}\right)^{2}\right]\right)^{\frac{1}{2}} .
$$

Arguing as before (see (4.42)) we get

$$
\lim _{n \rightarrow \infty} E\left[\left(Y_{n, 0}-W_{0}\right)^{2}\right]=0,
$$

proving (4.40). To get (4.33) we denote $\mathcal{B}(0)$ the $\sigma$-algebra generated by $g(0, \omega)$ and apply Jensen's inequality to obtain

$$
\mathbb{E}\left[W_{0}^{2}\right]=\mathbb{E}\left[E\left[W_{0}^{2} \mid \mathcal{B}(0)\right]\right] \geq \mathbb{E}\left[\left(\mathbb{E}\left[W_{0} \mid \mathcal{B}(0)\right]\right)^{2}\right] .
$$

By simple computation, taking in account that

$$
\mathbb{E}\left[\mathbb{E}\left[G_{1}\left(v^{+}, \omega, \Lambda_{n}\right)-G_{1}\left(v^{-}, \omega, \Lambda_{n}\right) \mid \mathcal{B}_{-1}^{\leq}\right] \mid \mathcal{B}(0)\right]=0,
$$

we have

$$
\begin{aligned}
& \mathbb{E}\left[W_{0} \mid \mathcal{B}(0)\right] \\
= & \mathbb{E}\left[\mathbb{E}\left[G_{1}\left(v^{+}(\cdot), \cdot, \Lambda_{n}\right)-G_{1}\left(v^{-}(\cdot), \omega, \Lambda_{n}\right) \mid \mathcal{B}_{0}^{\leq}\right]-\mathbb{E}\left[G_{1}\left(v^{+}, \omega, \Lambda_{n}\right)-G_{1}\left(v^{-}, \omega, \Lambda_{n}\right) \mid \mathcal{B}_{-1}^{\leq}\right] \mid \mathcal{B}(0)\right] \\
= & \mathbb{E}\left[\mathbb{E}\left[G_{1}\left(v^{+}(\omega), \omega, \Lambda_{n}\right)-G_{1}\left(v^{-}(\omega), \omega, \Lambda_{n}\right)|| \mathcal{B}_{0}^{\leq}\right] \mid \mathcal{B}(0)\right] \\
= & \mathbb{E}\left[F_{n} \mid \mathcal{B}(0)\right] .
\end{aligned}
$$

The lower bound (4.33) is proven.

Lemma 4.10. Let $U_{n}(a)$ be as in (4.30). For any $a>0$ and any $\delta>0$,

$$
\lim _{n \rightarrow \infty} \mathbb{P}\left[U_{n}(a) \geq \delta\right]=0 .
$$

Proof. By Chebyshev's inequality we have that

$$
\mathbb{P}\left[U_{n}(a) \geq \delta\right] \leq \frac{1}{\delta} \mathbb{E}\left[U_{n}(a)\right] .
$$

Next we show $\mathbb{E}\left[U_{n}(a)\right] \rightarrow 0$ for all $a>0$.

$$
\begin{aligned}
\mathbb{E}\left[U_{n}(a)\right] & =\frac{1}{\left|\Lambda_{n} \cap \mathbb{Z}^{d}\right|} \sum_{i=1}^{\left|\Lambda_{n} \cap \mathbb{Z}^{d}\right|} \mathbb{E}\left[Y_{n, i}^{2} 1_{\left\{\left|Y_{n, i}\right| \geq a \sqrt{\left|\Lambda_{n} \cap \mathbb{Z}^{d}\right|}\right]}\right] \\
& \leq \frac{1}{\left|\Lambda_{n} \cap \mathbb{Z}^{d}\right|} \sum_{i=1}^{\left|\Lambda_{n} \cap \mathbb{Z}^{d}\right|}\left(\mathbb{E}\left[Y_{n, i}^{2 q}\right]\right)^{\frac{1}{q}}\left(\mathbb{P}\left[\left|Y_{n . i}\right|>a \sqrt{\left|\Lambda_{n} \cap \mathbb{Z}^{d}\right|}\right]\right)^{\frac{1}{p}} .
\end{aligned}
$$


By Jensen inequality and definition (4.37) we have

$$
\mathbb{E}\left[Y_{n, i}^{2 q}\right] \leq \mathbb{E}\left[W_{0}^{2 q}\right],
$$

which is a bounded quantity for all $q \geq 1$ since (4.38). Applying Chebyshev inequality and arguing as before we have

$$
\mathbb{P}\left[\left|Y_{n, i}\right|>a \sqrt{\left|\Lambda_{n} \cap \mathbb{Z}^{d}\right|}\right] \leq \frac{\mathbb{E}\left[W_{0}^{2}\right]}{a^{2}\left|\Lambda_{n} \cap \mathbb{Z}^{d}\right|}
$$

which for all $a>0$ tends to 0 when $n \rightarrow \infty$.

Lemma 4.11. For $\Lambda \subset \mathbb{R}^{d}, 0 \in \Lambda$, we have

$$
\frac{\partial}{\partial g(0)} \mathbb{E}\left[F_{n} \mid \mathcal{B}(0)\right]=-\theta \mathbb{E}\left[\int_{Q(0)} v^{+}(x, \omega) d x \mid \mathcal{B}(0)\right]+\theta \mathbb{E}\left[\int_{Q(0)} v^{-}(x, \omega) d x \mid \mathcal{B}(0)\right],
$$

where $Q(0):=[-1 / 2,1 / 2]^{d}$. Furthermore,

$$
\mathbb{E}\left[\frac{\partial}{\partial g(0)} \mathbb{E}\left[F_{n} \mid \mathcal{B}(0)\right]\right]=-2 \theta m^{+} .
$$

Proof. We first give the idea by a formal computation.

$$
\begin{aligned}
& \frac{\partial}{\partial g(0)} \mathbb{E}\left[F_{n} \mid \mathcal{B}(0)\right]=\frac{\partial}{\partial g(0)} \mathbb{E}\left[G_{1}\left(v^{+}, \omega, \Lambda\right) \mid \mathcal{B}(0)\right]-\frac{\partial}{\partial g(0)} \mathbb{E}\left[G_{1}\left(v^{-}, \omega, \Lambda\right) \mid \mathcal{B}(0)\right] \\
= & \mathbb{E}\left[\frac{\partial G_{1}(v, \omega, \Lambda)}{\partial g(0)}|\left(v^{+}(\omega), \omega\right)+\underbrace{\frac{\partial G_{1}(v, \omega, \Lambda)}{\partial v(0)} \mid\left(v^{+}(\omega), \omega\right)}_{=0} \frac{\partial v^{+}(0, \cdot)}{\partial g(0)}| \mathcal{B}(0)\right] \\
& -\mathbb{E}\left[\frac{\partial G_{1}(v, \omega, \Lambda)}{\partial g(0)}|\left(v^{-}(\omega), \omega\right)+\underbrace{\frac{\partial G_{1}(v, \omega, \Lambda)}{\partial v(0)} \mid\left(v^{-}(0), \omega\right)}_{=0} \frac{\partial v^{-}(0, \cdot)}{\partial g(0)}| \mathcal{B}(0)\right] \\
= & -\theta \mathbb{E}\left[\int_{Q(0)} v^{+}(x, \omega) d x \mid \mathcal{B}(0)\right]+\theta \mathbb{E}\left[\int_{Q(0)} v^{-}(x, \omega) d x \mid \mathcal{B}(0)\right]
\end{aligned}
$$

where the terms are zero as $v^{ \pm}$are minimizers. The last equality is obtained since

$$
\frac{\partial G_{1}(v, \omega)}{\partial g(0)} \mid\left(v^{ \pm}(\omega), \omega\right)=-\theta v^{ \pm}(x, \omega) .
$$

Unfortunately, $v^{ \pm}$is not differentiable in the random field everywhere. Lipschitzcontinuity in the field would be sufficient, but this is difficult to derive from the Euler-Lagrange equation because of the lack of convexity of the associated functional. 
A rigorous proof follows from 4.7 after taking conditional expectations. Furthermore, by Theorem 4.1, we have

$$
\begin{aligned}
\mathbb{E}\left[\frac{\partial}{\partial g(0)} \mathbb{E}\left[F_{n} \mid \mathcal{B}(0)\right]\right]=-\theta \mathbb{E}\left[\mathbb{E}\left[\int_{Q(0)} v^{+}(x, \omega) d x \mid \mathcal{B}(0)\right]\right] \\
\\
\quad+\theta \mathbb{E}\left[\mathbb{E}\left[\int_{Q(0)} v^{-}(x, \omega) d x \mid \mathcal{B}(0)\right]\right] \\
=\theta\left[-m^{+}+m^{-}\right] \\
=-2 \theta m^{+}
\end{aligned}
$$

LEMMA 4.12. If

$$
\mathbb{E}\left[\left(\mathbb{E}\left[F_{n} \mid \mathcal{B}(0)\right]\right)^{2}\right]=0
$$

then $m^{+}=m^{-}=0$.

Proof. Denote $f(\omega(0)):=\mathbb{E}\left[-F_{n} \mid \mathcal{B}(0)\right]$. Set $s=\omega(0)$, Then (4.48) can be written as $\int f^{2}(s) \mathbb{P}(d s)=0$. By Lemma 4.11 and by bound (4.1) in Theorem 4.1 we have that $\left(1+C_{0} A \theta\right) \theta \geq f^{\prime}(s) \geq 0$ almost everywhere. This implies that $f(s)=0$ for $\mathbb{P}$ almost all points of continuity of the distribution $g(0)$. If $f(s)=0$ for $\mathbb{P}$ almost all points of continuity of the distribution $g$, then $f^{\prime}(s)=0$ for $\mathbb{P}$ almost all points of continuity of the distribution $g(0)$. But if $f^{\prime}(s)=0$ then from Lemma 4.11 we get $m^{+}=m^{-}=0$. $\square$

Proof of Theorem 2.2. By Lemma 4.2 there exists $C=C\left(C_{0}, A \theta\right)>0$ so that

$$
\left|G_{1}\left(v^{+}, \omega, \Lambda\right)-G_{1}\left(v^{-}, \omega, \Lambda\right)\right| \leq C|\Lambda|^{\frac{d-1}{d}} .
$$

Applying Theorem 4.8 we get the following lower bound on the Laplace transform of $F_{n}(\omega)$ defined in Definition 4.3:

$$
\liminf _{n \rightarrow \infty} \mathbb{E}\left[e^{t \frac{F_{n}}{\sqrt{\Lambda_{n}}}}\right] \geq e^{\frac{t^{2} D^{2}}{2}}
$$

where we denote $D^{2}($ see $(4.27))$ by

$$
D^{2}=\mathbb{E}\left[\left(\mathbb{E}\left[F_{n} \mid \mathcal{B}(0)\right]\right)^{2}\right] .
$$

It is immediate to realize that (4.49) and (4.50) contradict each other in $d \leq 2$ unless $D^{2}=0$. On the other hand when $D^{2}=0$, Lemma 4.12 implies

$$
m^{+}=-m^{-}=\mathbb{E}\left[\int_{\left[-\frac{1}{2}, \frac{1}{2}\right]^{d}} v^{ \pm}(x, \cdot) \mathrm{d} x\right]=0 .
$$

Now (4.5) implies that $P$-a.s. $v^{+}(x, \omega) \geq v^{-}(x, \omega)$ for all $x \in R^{2}$. This and (4.52) imply that $v^{+}(x, \omega)=v^{-}(x, \omega)$ a.s.

For each $n$ and for each $\omega \in \Omega$, by lower semicontinuity and coerciveness of the functional $G_{1}$, there exists at least one minimizer $w_{n}(\cdot, \omega)$ in $\min _{w \in H^{1}\left(\Lambda_{n}\right)} G_{1}\left(w, \omega, \Lambda_{n}\right)$. By (4.5) and the fact that $v^{+}=v^{-}$, the functions $w_{n}$ converge pointwise to a limit $u^{*}(x, \omega)$, and for the limit we have $u^{*}(x, \omega)=v^{+}(x, \omega)=v^{-}(x, \omega)$. The properties of 
the minimizer stated in 2.2 therefore follow from the corresponding properties of $v^{ \pm}$; see Theorem 4.1.

\section{Appendix A.}

The main tool to prove Lemma 4.8 is the following general result which we reported from [19]; see Theorem 3.2 and Corollary 3.1 of [19]. The correspondence to the previous notation is $\frac{F_{n}}{\sqrt{\left|\Lambda_{n} \cap \mathbb{Z}^{d}\right|}} \leftrightarrow S_{n}, \frac{Y_{n, i}}{\sqrt{\left|\Lambda_{n} \cap \mathbb{Z}^{d}\right|}} \leftrightarrow X_{n, i}$ and $\mathcal{B}_{n, i} \leftrightarrow \mathcal{F}_{n, i}$; see (4.20),

TheOREM A.1. Let $S_{n, i}, i=1, \ldots k_{n}$ be a double array of zero mean martingales with respect to the filtration $\mathcal{F}_{n, i}, \mathcal{F}_{n, i} \subset \mathcal{F}_{n+1, i} i=1, \ldots k_{n}$ with $S_{n, k_{n}}=S_{n}$, so that $S_{n, i}=\mathbb{E}\left[S_{n} \mid \mathcal{F}_{n, i}\right]$. It is assumed that $k_{n} \uparrow \infty$ as $n \uparrow \infty$. Denote

$$
\begin{gathered}
X_{n, i}:=S_{n, i}-S_{n, i-1}, \\
V_{n}=\sum_{i=1}^{k_{n}} \mathbb{E}\left[X_{n, i}^{2} \mid \mathcal{F}_{n, i-1}\right], \\
U_{n, a}=\sum_{i=1}^{k_{n}} \mathbb{E}\left[X_{n, i}^{2} \mathbb{I}_{\left\{\mid\left[X_{n, i}^{2} \mid>a\right\}\right.} \mid \mathcal{F}_{n, i-1}\right] .
\end{gathered}
$$

Suppose that

- for some constant $b^{2}$ and for all $\delta>0, \lim _{n \rightarrow \infty} \mathbb{P}\left[\left|V_{n}-b^{2}\right| \geq \delta\right]=0$,

- for any $a>0$ and for any $\delta>0$,

$$
\lim _{n \rightarrow \infty} \mathbb{P}\left[U_{n}(a) \geq \delta\right]=0,
$$

(Lindeberg condition)

then in distribution

$$
\lim _{n \rightarrow \infty} S_{n} \stackrel{D}{=} Z
$$

where $Z$ is a gaussian random variable with mean zero and variance $b^{2}$.

Acknowledgments. We would like to thank the anonymous referees for their careful reading and helpful comments.

\section{REFERENCES}

[1] M. Aizenman and J. Wehr, Rounding effects on quenched randomness on first-order phase transitions, Commun. Math. Phys., 130, 489-528, 1990.

[2] V. Berdichevsky, Distribution of minimum values of stochastic functionals, Netw. Heterog. Media, 3, 437-460, 2008.

[3] V. Berdichevsky, Distribution of minimum values of weakly stochastic functionals, in Homogenization, V. Berdichevsky, V. Jikov, G. Papanicolaou (eds.), World Scientific, 1999.

[4] L. Berlyand, O. Bruno and A. Novikov, Rise of correlations of transformation strains in random polycrystals, SIAM J. Math. Anal., 40, 1550-1584, 2008.

[5] A. Bovier, Statistical Mechanics of Disordered Systems. A Mathematical Perspective, Cambridge University Press, 2006.

[6] A. Braides and A. Piatnitski, Overall properties of a discrete membrane with randomly distributed defects, Arch. Ration. Mech. Anal., 189, 301-323, 2008.

[7] A. Braides and C. Zeppieri, Multiscale analysis of a prototypical model for the interaction between microstructure and surface energy, Interfaces Free Bound., 11, 61-118, 2009. 
[8] J. Bricmont and A. Kupiainen, Phase transition in the three-dimensional random field Ising model, Commun. Math. Phys., 116, 539-572, 1988.

[9] M. Cassandro, E. Orlandi and P. Picco, Typical configurations for one dimensional random field Kac model, Ann. Prob., 27, 1414-1467, 1999.

[10] M. Cassandro, E. Orlandi, P. Picco and M.E. Vares, Typical configurations for one dimensional random field Kac model: localization of the phases, Electronic J. of Probab, 20, 786-864, 2005.

[11] M. Cassandro, E. Orlandi and E. Presutti, Interfaces and typical Gibbs configurations for one-dimensional Kac potentials., Prob. Theor. Rel. Fields, 96, 57-96, 1993.

[12] G. Dal Maso and L. Modica, Nonlinear stochastic homogenization, Ann. Mat. Pura Appl., 144, 347-389, 1986.

[13] G. Dal Maso and L. Modica, Nonlinear stochastic homogenization and ergodic theory, J. Reine Angew. Math., 368, 28-42, 1986.

[14] N. Dirr, M. Lucia and M. Novaga, $\Gamma$ - convergence of the Allen-Cahn energy with an oscillating forcing term, Interfaces and Free Boundaries, 8, 47-78, 2006.

[15] N. Dirr, M. Lucia and M. Novaga, Gradient theory of phase transitions with a rapidly oscillating forcing term, Asymptotic Analysis, 60, 29-59, 2008.

[16] N. Dirr and E. Orlandi, Sharp-interface limit of a Ginzburg-Landau functional with a random external field, SIAM J. Math. Anal., 41, 781-824, 2009.

[17] L.C. Evans, Partial Differential Equations, Graduate Studies in Mathematics, AMS, 19, 1998.

[18] D. Gilbarg and N.S. Trudinger, Elliptic Partial Differential Equations of Second Order, Grundlehren der Mathematischen Wissenschaften, 2nd ed., Berlin, 1983.

[19] P. Hall and C.C. Heyde, Martingale Limit Theory and its Application, New York, Academic Press, 1980.

[20] G. Keller, Equilibrium States in Ergodic Theory, Londom Math. Society, Student Texts, 42, 1998.

[21] L. Modica, The gradient theory of phase transitions and the minimal interface criterion, Arch. Rational Mech. Anal., 98, 123-142, 1987.

[22] L. Modica and S. Mortola, Un esempio di $\Gamma^{-}$-convergenza, Bollettino U.M.I., 14-B, 285-299, 1997.

[23] E. Orlandi and P. Picco, Weak large deviations principle for one dimensional random field Kac model, Elect. J. Probab., 14, 1372-1416, 2009.

[24] E. Presutti, Scaling Limits in Statistical Mechanics and Microstructures in Continuum Mechanics, Springer Berlin Heidelberg, Series: Theoretical and Mathematical Physics, 2009. 